\title{
Effect of Rejection Performance on Hollow Fiber Composite Reverse Osmosis Membrane by Alcohols Additives
}

\author{
Xinghua Wang \& Wei Wang \\ Key Laboratory of Hollow Fiber Membrane Material and Membrane Process \\ Tianjin Polytechnic University \\ Tianjin Motian Membrane Engineering and Technology CO.Ltd. \\ Tianjin 300160, China \\ E-mail: star1232007@yahoo.cn
}

\begin{abstract}
The polyamide composite membranes were prepared from m-phenylenediamine aqueous and trimesoyl chloride hexane solution through interfacial polymerization technique on the polysulfone hollow fiber ultrafiltration supporting membrane. The effects of different types, different concentrations of alcohols additives on the properties of reverse osmosis composite membrane separation. The experimental results indicated that different types of alcohol additives on the retention properties of composite membrane is different; the certain concentration of alcohol additives can be retained in the membrane properties of the same circumstances, so that membrane water flux increased 5-10 times.
\end{abstract}

Keywords: Hollow fiber, Composite reverse osmosis membrane, Interfacial polymerization, Alcohol additives, Modification

\section{Introduction}

Reverse osmosis composite membrane is reverse osmosis membrane prepared by chemical methods such as interfacial polymerization, plasma polymerization, lamination, coating solution and impregnation and so on as the base film of ultrafiltration membrane. Hollow fiber reverse osmosis membrane is asymmetric membrane with self-supporting layer, whose shape is fibrous. The dense layer of it can be located not only on the outer surface of the fiber but also on the inner surface. A hollow fiber module prepares very simply, possesses small amplification and can provide a larger membrane area in the unit volume(Safrai,2008). A hollow fiber module would give a higher packing density than a tubular one and allow a less demanding pretreatment and maintenance than a spiral-wound one (McHarg,2004).Reverse osmosis composite membrane has been widely used in the desalination of salt water and seawater, and a great amount of industrial water has begun to use reverse osmosis technology to treat, so the development of high-performance reverse osmosis membranes has always been one of the focuses of membrane technology (Cadotte J E,1981;Petersen R ,1993)'. Thin hydrophilic composite layer obtained by membrane modification or adding different additives can improve membrane performance. Shi Qiuet al find the addition of phase transfer catalyst in aqueous solution makes the rejection rate and flux increased by $20 \%$ than the original membrane in the preparation of reverse osmosis membranes ( Shi Qiu, 2009). Under the same retention rate, the addition of hydrophilic additives (such as ethanol and phenol) in the aqueous phase can significantly increased flux, and the system wins the reverse osmosis composite membrane of high flux. Kuehne immerses the membrane into solutions containing various organic additives, and the flux of reverse osmosis composite membrane increases by 30\% to 70\%.(Mark A.2001) Jeong prepares reverse osmosis composite membrane using nano-particles of zeolite to make flux increase one time without affecting retention. (B.H. Jeong,2007). In this paper, polyamide reverse osmosis composite membrane is prepared by interfacial polymerization of m-phenylenediamine (mPD) as the aqueous phase monomer and trimesoyl chloride (TMC) as the organic phase monomer on the surface of polysulfone (PSF) hollow fiber ultrafiltration membrane as the base film. And adding different alcohols as additives in the amine solution discusses alcohols of polarity and swelling and other physical properties to the membrane to improve the rejection performance of it.

\section{Experimental}

\subsection{Chemicals and instruments}

Polysulfone (PSF) hollow fiber ultrafiltration membrane was obtained from Tianjin Motian Membrane Engineering Technology Co.,Ltd. (PR China), which is the inner diameter of 0.75mm and 20,000 MWCO. M-phenylenediamine (MPD), trimesoyl chloride (TMC), heptane, methanol, ethanol, propanol, isopropanol, n-butanol, isobutanol, butanol, tert-butyl alcohol and $\mathrm{NaCl}$ were analytical grade and used without further purification. 
Salt concentrations for permeation test were determined by electrical conductivity DDS-11A (Shanghai Leici Instrument, PR China). Membrane permeation tests were performed using self-made tester.

\subsection{Preparation of reverse osmosis composite membrane}

Composite membrane is prepared by interfacial polymerization. 15 of hollow fiber ultrafiltration membranes, whose length is about $1 \mathrm{~m}$, set as a bunch and rinse with water casting resin at both ends. Purge the interior of ultrafiltration membranes with air compressor. Then coat the interior of modules using $30 \mathrm{ml}$ aqueous solution of configured MPD, dry and coat with $30 \mathrm{ml} \mathrm{TMC} \mathrm{solution.} \mathrm{At} \mathrm{last,} \mathrm{dry} \mathrm{again} \mathrm{and} \mathrm{obtain} \mathrm{internal} \mathrm{pressure} \mathrm{hollow}$ fiber composite membranes.

\subsection{Characterization of reverse osmosis composite membrane}

The salt rejection and permeate flux of hollow fiber composite membranes were measured at $0.7 \mathrm{MPa}$ and room temperature with $500 \mathrm{mg} / \mathrm{L} \mathrm{NaCl}$ solution in a home-made evaluation device of membrane performance (Fig.1).

The permeation flux, F, is calculated as follows:

$$
F=\frac{V}{S \times t}
$$

where $\mathrm{V}$ is the total volume of solution permeated during the experiment, $\mathrm{S}$ the virtual membrane area and $\mathrm{t}$ is the operation time.

Rejection, $\mathrm{R}$, is defined by the following equation:

$$
R=\left(1-\frac{C_{p}}{C_{0}}\right) \times 100 \%
$$

where $\mathrm{C} 0$ and $\mathrm{Cp}$ are feed concentration and permeate concentration, respectively.

\section{Results and discussion}

\subsection{Effect of MPD concentration on the performance of composite membrane}

In Fig. 2, the effect of $\mathrm{mPD}$ concentration on composit reverse osmosis membrane were shown, and the concentration of TMC organic solution was $2 \mathrm{~g} / \mathrm{L}$.From Fig.2 we can see that with the increasing of mPD concentration, the Composite membrane flux reduced rapidly, then essentially the same .And the rejection of membrane Increased gradually, then remained stable. Mainly due to with the increasing $\mathrm{mPD}$ concentration of aqueous phase, the composite layer which formed at the interface was gradually thicker. And the effective thickness of the film increased, which made the flux fall and retention rate increase gradually. When the mPD concentration was too large, excess water phase monomer which no longer participated in polymerization reactions left in the hole of the hollow fiber membrane. The results showed that when the mPD concentration in aqueous phase was $0.5 \%$, the composite membrane had good separation performance, which had the highest retention rate of $98.2 \%$ and water flux of $1.46 \mathrm{~L} /(\mathrm{m} 2 \mathrm{~h})$.

\subsection{Effect of TMC concentration on the performance of composite membrane}

Figure 3 showed the effect of TMC concentration in the organic phase on performance of reverse osmosis membranes, mPD concentration in Aqueous phase was $0.5 \mathrm{wt} \%$. As can be seen from Figure 3, with the TMC concentration increasing in organic phase, the rejection of reverse osmosis composite membrane first increased, then the trend slow down and reached a stable value. At last the rejection reduced slightly. The overall flux showed upward trend. When the TMC concentration was $2 \mathrm{~g} / \mathrm{L}$, the rejection reached a maximum of $95.6 \%$. But the rejection decline at last due to the too much large concentration of TMC. This is because the functional layer of composite membrane play a decisive role on the performance of composite membrane (A L Ahmad,B,2005). The composite membrane which had uniform and dense function thin layer had a high retention rate. The increasing of TMC concentration in organic phase lead the increasing of the crosslinking density of the formation of functional layer by Interfacial polymerization. Functional layers of network-like structure was more dense. However, if the TMC concentration of the organic phase was too large, $\mathrm{MPD}$ will soon be run out because of excessive interfacial polymerization. So parts of the unreacted chloride would be transformed into carboxyl groups lose their activity. Resulted in water - oil interface forming loose polymer network. At this point membrane retention rates will be lower and flux increased.

\subsection{Effect of rejection performance reverse osmosis membrane by Alcohols additives}

Fig.4 shows the influnence of the RO membrane by the mathanol. As the methanol concentration increased,the salt rejection decreased while the flux first increase and then decrease. Methanol concentration of $1.2 \% \mathrm{wt}$ is the 
best time. Solubility parameters (E. A. Grulke,1989) are showed in Table 1 .Solubility parameters of methanol is 29.7MPa1/2 and solubility parameter of Polyamide composite membrane(S. M. Aharoni,1992)is $23 \mathrm{MPa} 1 / 2$, there is a gap. Therefore it is not because of swelling of change between the composite membrane performance. There is probably because methanol is highly volatile, the base film surface moisture removed soon, so that uniform coating phenylenediamine, the preparation of reverse osmosis membrane has good performance. Fig.5 shows that adding ethanol had little effect on the membrane, ethanol's solubility parameter $(26.0 \mathrm{MPa} 1 / 2)$ and the solubility parameters of polyamide composite membrane has a gap, it is not possible to improve the performance of composite membranes.Fig.6,7show that add Propanol,Isopropanol, Butanol, Butyl alcohol, Isobutyl alcohol can significantly increase water flux, salt rejection slightly. Water flux can increase 5-10 times. As can be seen from Table 1, their solubility parameters are very close to solubility parameter of Polyamide composite membrane.

\section{Conclusion}

(1) Monomer concentration on the interfacial polymerization of the composite reverse osmosis membrane performance is obvious. Selecting the appropriate concentration of monomer is prepared by interfacial polymerization key factor. The results show that: when the MPD concentration in aqueous solution is $0.5 \%$, TMC-heptane concentration is $2 \mathrm{~g} / \mathrm{L}$, the prepared hollow fiber reverse osmosis composite membrane is the best.

(2) The appropriate concentration of alcohol additive such as methanol, propanol, isopropanol, butanol, butyl alcohol, isobutyl alcohol can basically unchanged membrane retention rate, while the flux increased 5-10 times. Alcohols can change the performance of composite membrane is closely related with the solubility parameter.

\section{References}

A L Ahmad, B S Ooi. (2005). Properties-performance of thin film composite membrane:study on trimesoyl chloride content and polymerization time.[J]. J Membr Sci, 2005,255:67 77.

B.H. Jeong, E.M.V. Hoek, Y. Yan, A. Subramani, X. Huang, G. Hurwitz, A.K. Ghosh, A. Jawor. (2007). Journal of Membrane Science, 20072, 294(1-2): 1 7

Cadotte J E, King R S. (1981). Interfacial syntheses in the preparation of reverse osmosis membrane [J]. $J$ Macromol Scrchem, 1981, A15(5):727-755.

E. A. Grulke. (1989). Polymer Handbook, Third Edition. J. Brandrup and E. H. Immergut. Wiley, NewYork,1989.

Mark A. Kuehne, Richard Q. Song, Norman N. Li, Robert J. Petersen. (2001). Environment Progess, 2001, 20(1): $23 \sim 26$

McHarg, J. R. Turby. (2004). West coast researchers seek to demonstrate SWRO affordability [J]. Desalination \& Water Reuse, 2004. 14(3): 10-18.

Petersen R J. (1993). Composite reverse osmosis and nanofiltration membranes [J]. J Membrane Sci, 83(1993) 81-150.

S. M. Aharoni. (1992). The solubility parameters of aromatic polyamides. J. Appl. Polym. Sci., 1992, 45, 813.

Safrai , I., A. Zask. (2008). Reverse osmosis desalination plants -marine environmentalist regulator point of view [J]. Desalination, 2008. 220(1-3): 72-84.

Shi Qiu, Liguang Wu, Lin Zhang, Huanlin Chen,Congjie Gao. (2009). Journal of Applied Polymer Science, 2009,112(4): 2066 2072 
Table 1 . The solubility parameters of alcohols

\begin{tabular}{|l|l|l|l|l|}
\hline Name & Methanol & Ethanol & Propanol & Isopropanol \\
\hline Solubility parameter $(\delta) \mathrm{MPa}^{1 / 2}$ & 29.7 & 26.0 & 24.3 & 23.5 \\
\hline
\end{tabular}

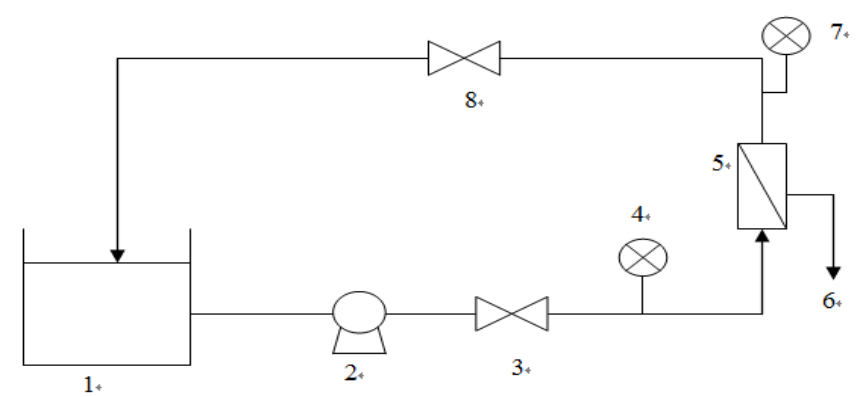

1 liquid tank, 2 diaphragm pump, 3 import valve, 4 import pressure gauge, 5 reverse osmosis membrane module, 6 penetration liquid, 7 export pressure gauge, 8 export valve.

Figure 1. Reverse osmosis schematic drawing of the experimental system used in this study

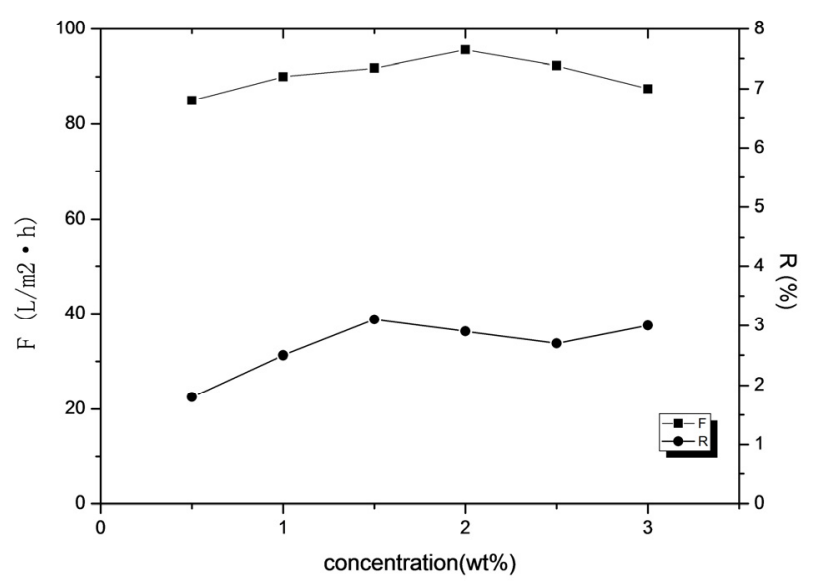

Figure 3. Effect of TMC concentration on salt rejection and water flux of the resulting membrane

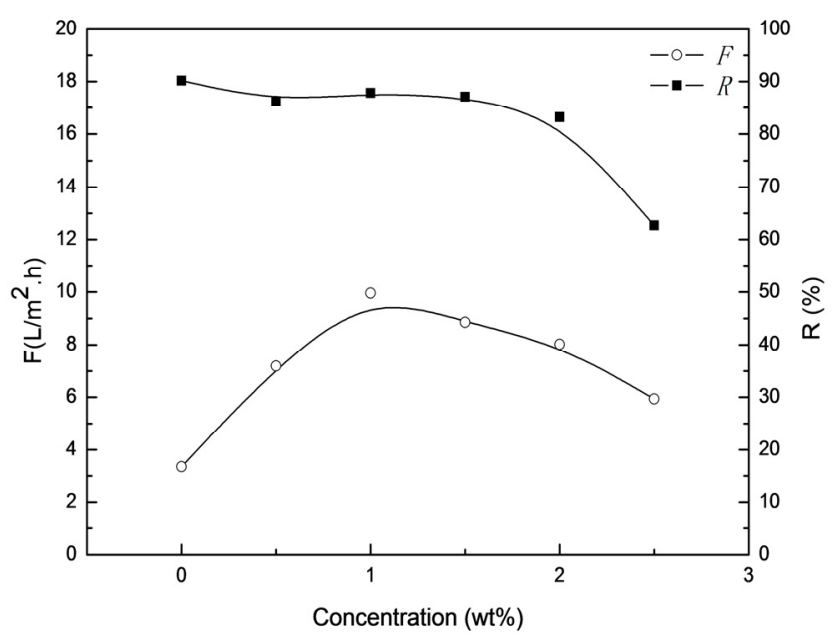

Figure 4. Effect of methanol concentration on salt rejection and water flux of the resulting membrane 


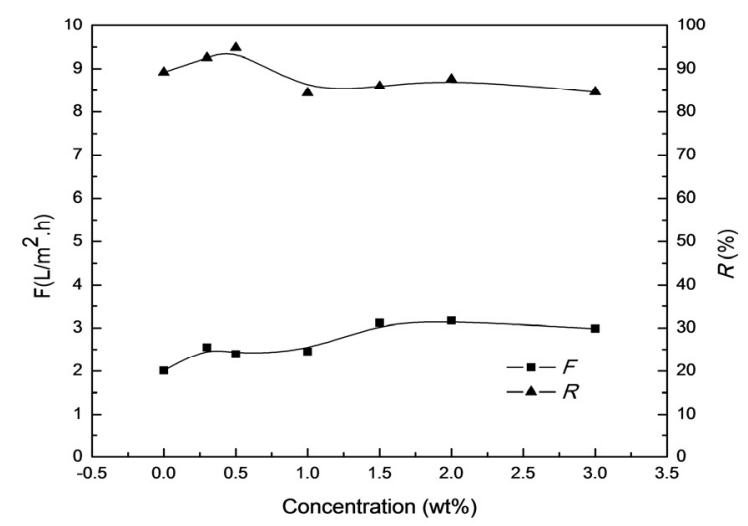

Figure 5. Effect of ethanol concentration on salt rejection and water flux of the resulting membrane

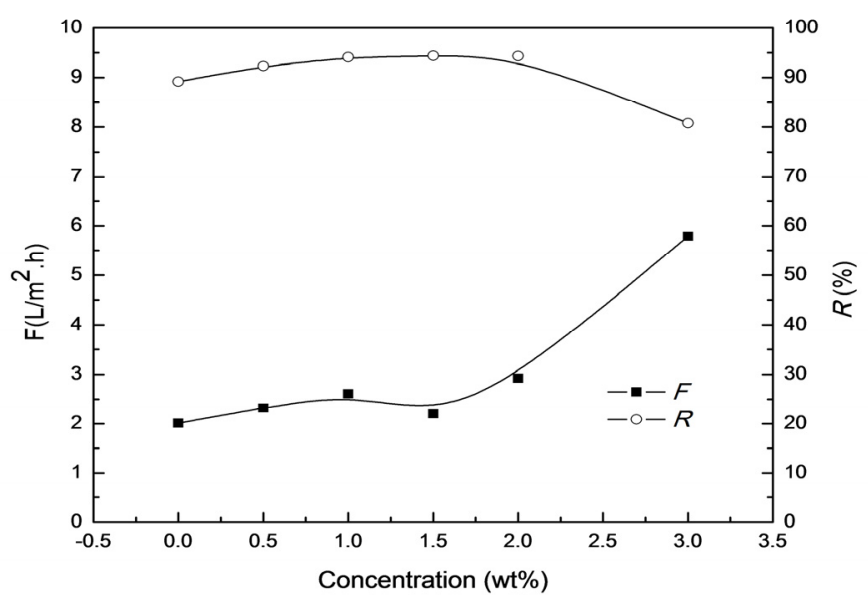

Figure 6. Effect of propanol concentration on salt rejection and water flux of the resulting membrane

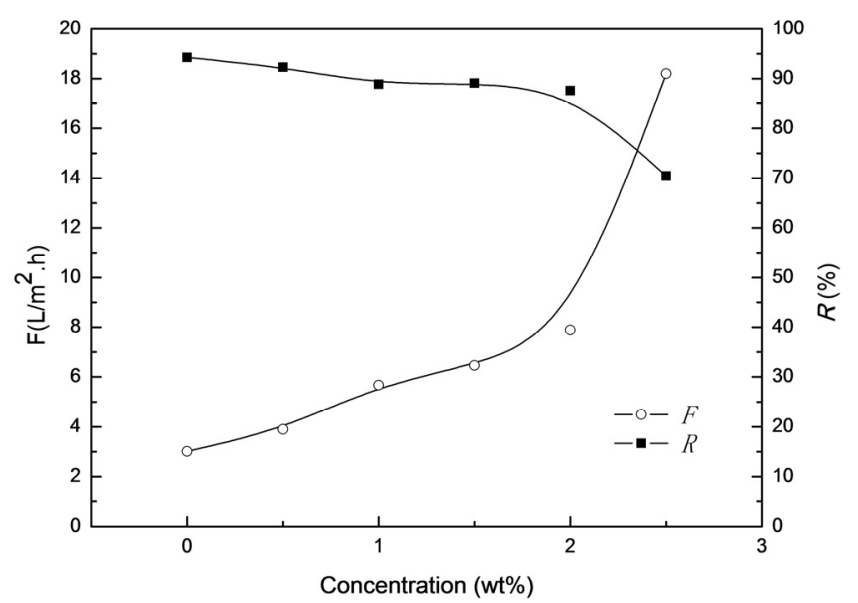

Figure 7. Effect of isopropanol concentration on salt rejection and water flux of the resulting membrane 